\title{
El discurso femenino de resistencia frente a la violencia dictatorial en Carne de Perra de Fátima Sime
}

\author{
The feminine discourse of resistance against the \\ dictatorial violence in Fátima Sime's Carne de perra
}

\author{
Nicole Muñoz Albornoz ${ }^{1}$ \\ Estudiante Magíster Universidad de Chile \\ jvt2301@gmail.com
}

\begin{abstract}
SÍNTESIS
En el presente trabajo, el objetivo principal es develar las implicancias de las estrategias textuales desplegadas por la enunciante, para hacer frente a la violencia ejercida sobre ella, tanto en el marco de la dictadura como de la postdictadura chilena. Para estos efectos, la argumentación se articula en tres ejes centrales. El primero de ellos relativo a la reconstrucción de una memoria quebrada, producto de las condiciones postraumáticas. El segundo aborda las prácticas de violencia, considerando los matices que éstas adquieren cuando la agredida es una mujer $y$, en este sentido resulta de especial interés, el juego de inversión de roles que se da entre víctima y victimario en la novela. Finalmente, en un último apartado, se indagará en relación al posicionamiento de estos discursos individuales marginales en el espacio público, mediatizado por una dictadura o postdictadura y, las consecuencias de esto frente a los discursos hegemónicos.
\end{abstract}

\begin{abstract}
In the present study, the main objective is to uncover the implications of the textual strategies deployed by the enunciating voice to confront violence exercised against her, both under the Chilean dictatorship and post-dictatorship. For these purposes, the argument is based on three main axes. The first one aims to the reconstruction of a broken memory, which is a product of post-traumatic conditions. The second one deals with the practices of violence, considering the nuances that they acquire when the aggression is towards a woman and, in this sense, is of particular interest the role reversal game that occurs between the
\end{abstract}


victim and the victimizer. Finally, in a final section, we will explore the positioning of these marginal individual discourses in the public space, mediated by a dictatorship or a post-dictatorship, and the consequences of this in front of the hegemonic discourses.

Palabras claves: Memoria, violencia, género, estrategias textuales, Chile, dictadura/postdictadura.

Keywords: Memory, violence, gender, textual strategies, Chile, dictatorship/postdictatorship.

\section{Reconstrucción de la memoria}

Durante los últimos años, el estudio de la memoria ha adquirido mucha fuerza en los países del Cono Sur, especialmente en la línea de los Estudios Culturales y, en el marco de la reflexión postdictatorial. En este sentido, autoras como Nelly Richard en Residuos y Metáforas (1998) y Elizabeth Jelin en Los Trabajos de la Memoria (2002), han dado cuenta de esta teorización en torno a la memoria en América Latina, tanto en sus trabajos de compilación como de reflexión propia.

En el caso de Richard (1998, 15), la elaboración de la memoria se inserta en la idea de lo residual, entendiendo por ello la existencia de elementos no integrados a las jerarquías discursivas, o bien considerados como secundarios dentro de las mismas y, cuya validación permitiría el cuestionamiento de los discursos oficiales. En este marco, la crítica enfatiza la relevancia del residuo como huella que puede productivizar el ejercicio de la memoria, atendiendo a su vez, al posicionamiento de estos residuos desde el espacio subalterno y marginalizado por el discurso oficial, como dice Richard:

La idea de lo residual como el modo en que lo secundario y lo nointegrado son capaces de desplazar la fuerza de la significación hacia los bordes más desfavorecidos de la escala de valores sociales y culturales, para cuestionar las jerarquías discursivas desde posiciones laterales y descentramientos híbridos $(1998,16)$.

Así la autora vincula esta noción de memoria con la experiencia postdictatorial, dando cuenta de un dilema melancólico que se disputa entre el recordar y el olvidar, provocando fenómenos como el 
enmudecimiento del sujeto o la sobreexcitación del mismo. En este sentido, la inscripción de la memoria está fuertemente ligada a la "escena de producción de lenguajes" (Richard, 1998, 55), que busca de alguna manera, restaurar el sentido a través de la narrabilidad del recuerdo como testimonio. Sin embargo, para Richard (1998, 72), este testimoniante suele estar fragmentado ante un duelo irresoluto que le impide un relato lineal y, en cambio, posibilita un resucitar trizado del pasado como actualidad viva.

Otro de los fenómenos que adquiere importancia para Richard en Políticas y Estéticas de la memoria (2006) es el concepto del habla mutilada (11), ya que la voz enunciante vivió durante largo tiempo en silencio, sin posibilidad de expresión más que simbólica o metafórica, por lo que para volver a manifestarse, requiere de recursos que le permitan insertarse en los nuevos discursos argumentativos generados por las estrategias transicionales. En este marco, la escritura y el ejercicio de la memoria se asocian a un discurso fragmentario y entrecortado, ya que si bien lo que se pretende es dejar en evidencia a las políticas de borradura de la huella, es decir, de borrón y cuenta nueva, al mismo tiempo, el enunciante no encuentra otras formas de expresión de su relato, que no estén vinculadas con la narración fragmentaria.

Por su parte, Elizabeth Jelin en Los Trabajos de la Memoria $(2002,2)$ entiende la memoria en tres sentidos. En primer lugar, como un proceso subjetivo, en la medida que ancla en experiencias fundamentalmente personales que además se expresan de manera simbólica. En segundo término, la visualiza como un objeto de disputas, ya que los procesos de transición política y social, la han utilizado como recurso que legitima la condena a los crímenes cometidos, pero a su vez, en algunos casos, no se considera su verosimilitud como prueba en actos de reparación hacia las víctimas. Finalmente, en tercer lugar, da cuenta de la necesidad de historizar las memorias, reconociendo su incidencia tanto en el pasado como en el presente. De esta manera, la reconstrucción de la memoria en el caso del proceso postdictatorial, se asocia también, a la reconstrucción de una identidad individual y colectiva posterior a la violencia y al trauma acontecidos.

En este marco de reflexión, es importante entender que "ubicar temporalmente a la memoria significa hacer referencia al espacio de la experiencia en el presente" (Jelin, 2002, 3), aunque esta incorporación 
no es estática sino dinámica, ya que las experiencias a veces se incorporan en momentos posteriores. En efecto, la relación del sujeto con ese pasado corre el riesgo de configurarse como un permanente retorno (Jelin, 2002, 14), porque puede quedarse anclado en la narración de su experiencia, en la constante repetición de ésta y en la imposibilidad de proyectarla en el otro.

Otra de las situaciones en las que Jelin $(2002,27)$ hace hincapié, es en la forma que adquiere el acontecimiento rememorado en la escritura, ya que es ésta la que permite al sujeto construir un relato comunicable y coherente. Sin embargo, este relato se inserta en lo que entendemos como narrativa subjetivada (Jelin, 2002, 87), porque da cuenta de una verdad que no se enmarca necesariamente, en nuestros propios criterios de verdad

Otra autora que reflexiona en torno a estas problemáticas en $\mathrm{El}$ Arte de no Olvidar: Literatura Testimonial en Chile, Argentina y Uruguay entre los 80 y los 90 (2006) es Nora Strejilevich. Para ella, el problema no es que esta narrativa sea incapaz de evidenciarlo todo, sino que la dificultad está en creer que efectivamente, podemos exigir una entrega de lo vivido de la forma en la que estamos acostumbrados, por ejemplo, por los medios de comunicación que pretenden configurar narraciones acabadas (10). Este carácter no lo encontramos en los relatos de postdictadura. En éstos, el sujeto puede haber olvidado o bloqueado partes de la vivencia, tanto voluntaria como involuntariamente, e incluso, en la mayoría de las oportunidades, su memoria fragmentada, no le permite dar cuenta de sus recuerdos, como una totalidad organizada de manera lineal (19). Ello supone un pacto de lectura que resulta bastante más complejo que el que se da en un simple relato de ficción, porque el lector espera una determinada forma de presentación de la verdad, mientras quien narra debido al trauma, es incapaz de exhibirla de manera lineal y el fragmento aparece como única posibilidad de dejar que el recuerdo se filtre en la narración en palabras de Strejilevich: “En el escrito la página en blanco es, textualmente, el espacio donde el evento se inscribe por vez primera y la memoria actúa creando un texto donde elaborar su horizonte desde la subjetividad, o donde el narrador la reinscribe para que ésta recupere su estatuto" (17).

Por lo tanto, una arista que surge es el cuestionamiento en torno a la credibilidad de estas narraciones, ya que el lector habitual 
maneja una terminología y unas expectativas que difieren del modo en el que se entrega la información en este discurso. Por lo tanto, el conflicto se da en relación a qué es la verdad y cómo debe transmitirse (Strejilevich, 10). Sin embargo, más allá de este aire de irrealidad (Strejilevich, 12) que rodea de manera inherente a estos relatos, lo importante es asumir que, especialmente en la reflexión postdictatorial, la inscripción de la memoria se basa en la subjetividad del escribiente que, si bien está teñida por un deseo de verosimilitud, a la vez debe asumir su propia imposibilidad de dar cuenta de manera acabada de un referente. De esta forma, el narrar en y por el recuerdo, se convierte en la tarea de juntar fragmentos que permitan elaborar algún sentido (Strejilevich, 19).

Otro de los autores que se ha preocupado de estas reflexiones, desde una perspectiva sociológica es Tomás Moulian en Chile Actual: Anatomía de un mito (1997). En este texto plantea que lo más importante es dar cuenta del resultado que tuvo el proceso dictatorial en la reconstrucción del país, entendiendo que la reconstrucción que se propone la dictadura, implicó borrar todo lo avanzado en el gobierno de Allende y construir un "nuevo país" desde nuevas bases, que tenían un carácter excluyente y no democrático. En este sentido, Moulian asume que es inevitable el influjo de los cambios que produjo la dictadura como anulación no solo de la cultura en la sociedad, sino también del sujeto mismo. Así, más que abogar por un modelo generalizado, se enfoca en destacar las experiencias individuales que logran posicionarse, de alguna manera, como muestras de lo colectivo, dando lugar a "resonancias individuales, que son ecos de experiencias colectivas, pero resignificados por psiquis particulares" (31).

De acuerdo a lo anterior, podemos ver que la reflexión en torno a la memoria entendida en un proceso dictatorial y postdictatorial, implica poner en juego al menos dos factores. En primera instancia, el fluir del recuerdo y, en segundo lugar, la plasmación de éste en la escritura. Esto último contiene un cuestionamiento en relación a la verdad, ante un relato que asume su propia imposibilidad de abordar a cabalidad los hechos acontecidos.

En este marco de reflexión que propongo, podemos identificar una tendencia de presentización del pasado, es decir, una necesidad de los enunciantes de contar sus experiencias vividas en situaciones 
límites, como la tortura y la violación a los derechos humanos, con intenciones que van desde la catarsis hasta la legitimación del testimonio como prueba de verdad. Sin embargo, el texto que convoca este trabajo, no es un relato real, sino que ficticio, en la medida que la enunciante no vivió los hechos que relata, sino que su afán es una elaboración ficcional de un caso que pudiera haber ocurrido en plena dictadura chilena. De esta manera, la justificación del análisis de este texto desde categorías propuestas por el ejercicio de la memoria, pasa tanto por la temática que es abordada en éste, como por la apropiación narrativa que se realiza de las estrategias textuales que se utilizan en relatos que rescatan el ejercicio de la memoria.

Sin embargo, antes de entrar directamente en el análisis, me parece pertinente hacer un breve resumen de la trama de la novela. Fátima Sime, autora del texto Carne de Perra (2009), nos cuenta la historia de una enfermera que fue llevada a un centro de tortura por motivos indefinidos, y que luego fue secuestrada de este lugar por uno de sus torturadores. De esta forma, nuestra protagonista es arrancada del "sistema de tortura" y llevada a una ilegitimidad aún mayor, si es que pudiera haberla. Así, la enfermera termina siendo confinada en un departamento en el que permanentemente es visitada por su torturador y violador, desarrollándose una relación, por decir lo menos, particular entre ellos, ya que avanzada la novela, podemos decir que nos encontramos frente a un "síndrome de Estocolmo", porque si bien ella puede escapar -no tiene vigilancia permanente y posee llaves del departamento-, no lo hace.

Así, el lugar desde el que se posiciona la sujeto para realizar su narración, permite un análisis de las estrategias de reconstrucción de la memoria, ya que asistimos a un relato que se despliega desde un tiempo presente, en el que nuestra protagonista ha vuelto del exilio, trabaja en un hospital y, un día cualquiera descubre que uno de sus pacientes es su torturador, quien está aquejado por un cáncer terminal. Desde ahí, se gatillan todos sus recuerdos y, por lo tanto, se inicia la rememoración de todas las violaciones cometidas y, la reconstrucción narrativa de éstas.

En este contexto, la primera situación que salta a la vista es el cambio temporal que se produce en la narración, porque existe una alternancia entre el relato que cuenta la tortura a la que estuvo sometida en su juventud, que aparece en tercera persona y, la narración 
de esta nueva tortura a la que es sometida en el presente, que se cuenta en primera persona. Este hecho, puede entenderse desde la necesidad de negación y disociación del sujeto torturado, ya que en palabras de Margarita Díaz:

Expuestas a un poder arbitrario extremo, en muchas ocasiones sólo pudieron resistir usando mecanismos de defensa, como la negación $[. .$.$] de disociación, como si estuvieran mirando desde$ afuera un cuerpo violado y ultrajado, pero sin sentirlo como el propio o incluso con episodios psicóticos transitorios, durante los cuales el delirio las transportaba fuera de esa institución (17)

De esta manera, una estrategia textual que podemos ver como una maniobra estética, esconde un trasfondo relevante, ya que tal como lo propone la profesora María Olga Ruiz, el torturado y el violado, es anulado como sujeto, en la medida que se produce una objetualización del cuerpo agredido y, por lo tanto se vuelve una entidad sin voz, un ser marginal que necesita de "otro", aunque éste sea ficticio, para expresarse.

La presentización del recuerdo llevada a cabo por la protagonista, está gatillada por una situación límite, que es la reaparición del torturador en su vida, lo que trae consecuencias en su escritura, ya que asistimos a una permanente confusión de los hechos relatados: "No recuerda haberle mencionado el detalle de la higuera en la casa de sus padres. Pero hay cosas que se le pierden, se le confunden. No confía ni en sí misma. A veces no distingue los recuerdos de los sueños" (Sime 32). De esta manera, resulta pertinente evidenciar las secuelas de la tortura en el acto de narrar, ya que existe una compleja relación entre la tortura y su representación, en palabras de Idelber Avelar:

Los testimonios de presos políticos sometidos a la tortura, al enfrentarse con el problema de la traducción de su experiencia al lenguaje, inevitablemente dejan de manifiesto los límites de toda su representabilidad [...] Uno de los efectos calculados de la tortura es hacer de la experiencia una no experiencia, negarle a ella una morada en el lenguaje (176). 
Otro de los efectos que se evidencian en el relato, es el bloqueo de la memoria, ya sea de hechos que de manera conciente no se quieren relatar, como de otros que parecen haberse borrado: "Cuando se me atravesó la misma sensación de muerte de la noche anterior, y el golpeteo de la sangre en la sien izquierda se hizo insoportable. No era capaz de referirme a él ni con los pensamientos" (Sime 22).

Además debemos considerar que la presentización del recuerdo de la situación límite, suele estar gatillada por otra situación límite, como lo es el descubrimiento del torturador como paciente:

Era un olor dulzón a rosas [...] De pronto recordé el rosedal que había en medio de la plaza, en Limache, y me vi de pequeña, con mi familia, paseando. Pero qué estoy haciendo, pensé. El azar me había traído de vuelta a un enfermo. ¿Cuánto le quedaría de vida? ¿Tres meses? ¿Seis? ¿Un año, a todo reventar? Aspiré de nuevo el olor de las rosas. La cara de mi padre, la de mi madre, la de esa niña corriendo feliz en la plaza, se perfilaron en mi memoria con la nitidez de una foto (Sime 36).

De esta manera, el recordar surge, tal como lo propone Loreto Rebolledo, como una reconstrucción en los marcos del presente, una reconstrucción que debe asumir sus falencias y desencuentros.

\section{Prácticas de la violencia y género}

En este apartado me propongo reflexionar en torno a la problemática de género en el contexto de la dictadura y postdictadura, relevando algunas temáticas que las políticas de olvido han tratado de invisibilizar. En primer lugar, me gustaría destacar que la implantación de la dictadura implicó, en términos discursivos, un importante énfasis en la ya instaurada lógica del patriarcado. En este sentido, el discurso hegemónico dictatorial equiparó el accionar del régimen frente a sus opositores con las dinámicas ya existentes en la sociedad del hombre hacia la mujer. En palabras de Jelin:

Los rituales del poder en el escenario público (saludos militares, desfiles, etc.) tenían un carácter performativo, en el que se desplegaba sin matices la dualidad entre el actor/poder masculino por 
un lado y la pasividad/exclusión feminizada de la población o audiencia por el otro $(2011,1)$

Por lo que se evidencia una extensión de los roles asignados a cada género, a la convivencia de la sociedad.

En este marco resulta interesante dar cuenta de los distintos roles por los cuáles las mujeres eran detenidas y torturadas, ya que si bien algunas fueron detenidas por su participación como militantes de algún partido u organización de la resistencia, es decir, aludiendo a su rol político que además transgredía su "ser mujer", en su mayoría, las mujeres fueron detenidas por estar ligadas, ya sea familiar o afectivamente, a un militante. En este sentido, la mujer es utilizada para que el militante se entregue o para obtener información de su paradero. De esta manera, el régimen no solo castiga a la mujer que sobrepasa los límites asignados a su género, sino que también la vuelve un objeto de chantaje para sus propósitos.

En lo que respecta a las mujeres que no fueron detenidas, ellas también vivieron una transgresión de su rol, en la medida que ante la ausencia del padre proveedor tuvieron no solo que asumir esa labor, sino que aprender a conjugarla con la búsqueda del detenido, en este sentido

... dos tipos de acciones "típicamente femeninas" se dieron en ese contexto: en la escena pública, la creación de organizaciones de derechos humanos ancladas en el parentesco con las víctimas directas. En el ámbito privado, se trató de la lucha por la subsistencia familiar y la adaptación o cambio en función de las nuevas circunstancias (Jelin, 2010, 2).

En este marco la violencia ejercida durante la dictadura chilena sobre la sociedad, pasó desde la desarticulación de las redes existentes entre las personas, hasta la tortura individualizada en campos de detención clandestinos. De esta última es de la que me haré cargo en este apartado, ya que como sabemos, a fin de cuentas, el texto relata la historia de una mujer torturada.

La tortura se manifiesta de dos maneras claras. En primer lugar está la tortura física, que en el caso de las mujeres, estuvo fuertemente asociada a la violencia sexual y a la denigración de ésta 
aprovechándose de su condición de mujer. De esta manera, el sujeto masculino ostenta su poder no solo como dominador frente al dominado, sino que también como hombre sobre la mujer:

Ahora ¡quítate el suéter! ¡No! La falda no. La quiero en falda y sostén, mi reina. Date vueltas. ¡Más! [...] Está sentado frente a la mesa con una botella de whisky en la mano y se ha bajado el cierre del pantalón. ¡En cinco minutos quiero a este muchacho en posición firme! ¿Oíste? (Sime 54).

En consecuencia, debemos considerar la temática del género y de la sujeción femenina, ya que la fuerza de la violencia sexual pasa precisamente por la instalación del imaginario de la mujer, como una sujeto débil, desamparada y que no puede defenderse por sí misma. Así, el hombre se posiciona como a quien hay que obedecer: “¿Tendría que importarme lo que a ti te gusta? Hoy no quiero enojarme ¿sabe? Le tengo una sorpresa, así que ¡ya! ¡Se me baja del auto! Ella obedece" (Sime 5) y complacer: "Se monta sobre ella. De un solo tirón le arranca la blusa. Le saca el sostén [...] El hombre toma el plato de la mesa, lo da vuelta encima, se lo restriega en la piel desnuda [...] Así le gusta comer al Príncipe" (Sime 20). Sin embargo, lo más importante es la posesión del hombre sobre la mujer, ella en su calidad de torturada es doblemente vista como objeto y no como sujeto: "Eso es lo que pareces ahora. Que no se te olvide: ¡mi muñeca!" (Sime 48).

Esta situación de sujeción de la mujer en la sociedad que se ve acrecentada con la tortura, tiene consecuencias tales como la naturalización de la violencia, tal como lo expone Carolina Herrera, las mujeres torturadas que sobrevivieron el régimen, suelen considerar que la violencia que se ejerció sobre ellas es menor, por ejemplo a la que se tuvo con los desaparecidos y, en este sentido, durante mucho tiempo las violaciones sexuales fueron un secreto a voces de los atentados cometidos por el régimen, ya que frente a otras problemáticas mayores como los desaparecidos o los muertos, las mujeres consideraban sus violaciones como un mal menor.

En segundo lugar, surge la tortura psicológica, ya que existió un chantaje permanente a los torturados con sus familias: "Va colocando 
las fotos en fila. ¡Qué bonita familia! Hay que cuidar que a esta familia no le ocurra nada" (Sime 9).

Por lo tanto, es necesario indagar en las secuelas de la violencia sexual en las mujeres, ya que surgen temáticas como las consecuencias psicológicas, por ejemplo, a nuestra protagonista se le diagnostica crisis de pánico y delirio de persecución en el exilio. Pero también, está la dificultad de relacionarse con otros, de desarrollar lazos reales de confianza, situación que Olga Grau explica a través de su concepto de lo erógeno herido:

Cuando pienso en lo erógeno herido, lo pienso en un doble sentido: como lo genital, lugar de ensañamiento preferente en la tortura calculada, planificada, y como lo vincular o vinculante. Lo dañado es lo erógeno, la posibilidad de constituir lazos confiables, de articulación de espacios comunitarios que se sostengan en el tiempo. Las zonas erógenas de la sociedad, la piel social, los umbrales de la sensibilidad, la confianza de las personas de pertenecer a una comunidad, a un proyecto común, se dañaron tan fuertemente, que una de las características predominantes de nuestra cultura es la desconfianza, la distancia, el descompromiso, la falta de pasión (25).

Otra de las problemáticas que se despliegan desde la tortura a mujeres, es la concepción que tienen éstas de su cuerpo: “La sensación de que mi cuerpo era solo una carcasa, mi alma un vacío desolado, no se apartaba de mí. ¿Por qué buscar hombres para llenar ese abismo?" (Sime 38), lo que nos lleva a repensar la relación que tiene el individuo con la materialidad de su cuerpo, ya que siguiendo a Raquel Olea la escritura de memoria suele hacer alusión al cuerpo porque es éste el que sufre, es la carne la torturada, pero a la vez, este cuerpo pertenece a un sujeto, a un alma que también sufre. Además, desde esta concepción del cuerpo surge también la problemática de la relación de la mujer torturada con los hombres: "Ni siquiera recordaba la cara de los tipos con que me acostaba. Mi modo de relacionarme con los hombres no era algo que me enorgulleciera, simplemente no podía evitarlo" (Sime 37) y en este marco, la imposibilidad de dejar atrás al torturador porque dejó su huella, su marca en el cuerpo, en la materialidad del ser: "Mi cuerpo no estaba convencido, tenía su propia memoria y seguía prisionero. En cada poro que se 
estimuló, en la humedad, en la congestión, en la turgencia, continuaba la marca del Príncipe. Junto a mi amante, me penetró también él" (Sime 41).

De esta forma, la violencia sexual genera un espacio difuso de temáticas que la mujer no consigue resolver. En este sentido, resulta de especial interés problematizar el síndrome de Estocolmo, ya que nuestra protagonista divaga y cuestiona permanentemente el carácter de su relación con el torturador: "Él era el amo, mi amo. Me producía terror. También me excitaba. Una mezcla que no lograba entender. Estaba presa. ¿De él? No. Más que de él, de mi apego a la vida" (Sime 51). Mientras el torturador propiciaba espacios y diálogos para confundirla:

Así no más soy yo, y a usted le está gustando. Dígame: "Me gustas así como eres". Ella: Sí, me gustas. Él: Sabe que más, usted también me gusta a mí. No es porque me la puedo comer como yo quiera. No, señor. Hay otras cosas por ahí... ¿Sabe por qué me gusta usted? Ella: ¿por qué? Él: Porque sí no más (Sime 56).

Esta situación nos lleva a cuestionar el fin de la resistencia del torturado frente al torturador, ya que para Paulina Gutiérrez, una reacción común es acabar en algún momento con la resistencia y desarrollar un comportamiento pasivo o bien disociado a fin de sobrevivir a la situación. Este mecanismo puede ser reinterpretado con posterioridad por la propia víctima como si se hubiese tratado de una suerte de complicidad y transformarse en una culpa que se revierte sobre ella.

Finalmente, abordaré la problemática de la inversión de roles, ya que la protagonista tiene la posibilidad de pasar de ser torturada a ser torturadora. En este sentido, la reaparición del torturador en una condición de vulnerabilidad le permite vengarse:

Esta noche, susurré, va a ser una noche de juegos. De esos juegos que tanto te gustaban a ti y a tu muñeca. Los recuerdas, ¿verdad? [...]Tomé la sonda y la retiré. ¿Te gustan mis juegos? Sentí en la mejilla el sudor de su cara. Esperé un poco. Él abría, en cada inspiración, cada vez más la boca. Cuando sus labios tomaron un leve tinte oscuro, volví el oxígeno a su lugar [...] Palpé la punta de su 
pene, tomé la sonda que recolectaba su orina y la cerré con una pinza (Sime 110).

Así, su tortura está mediada por la orden permanente del paciente que le pide que lo mate: "El Príncipe se creía aún con poder sobre mí. Quería obligarme a que yo pusiera fin a su dolor [...] Una enfermera es el amo. Es mi territorio. Esta vez yo tengo el poder" (Sime 57), pero la pregunta es ¿quién tortura a quién?, ¿quién tiene el poder? Este cuestionamiento toma más fuerza con la muerte del torturador que efectivamente es propiciada por ella:

No creas que estoy haciendo esto por ti, le dije, lo hago por mí, porque, sabes, yo tengo sentimientos, aunque no lo creas. Ni se inmutó. Te lo voy a pasar por la sonda de la morfina, agregué, vas a sentir que te entra vidrio molido en la vena por unos segundos, sólo van a ser unos segundos [...] De pronto, como si ya no tuviera más que decir, el índice quedó estático y fue cayendo, lentamente sobre la cama. Todas las máquinas empezaron a sonar (Sime 122).

En consecuencia, podemos observar que la violencia sexual ejercida durante la dictadura tomó especiales matices cuando se ejerció contra una mujer, ya que ésta reconstruye su identidad desde su condición de torturada: "La torturada, me creí la torturada heroica, la prisionera política que había sobrevivido, la víctima” (Sime 85).

\section{Posicionamiento del discurso marginal}

Un primer acercamiento a esta problemática, que debemos considerar, es que el discurso de la mujer torturada, tal como lo planteé anteriormente, es un discurso doblemente marginal, ya que es relegado tanto por la condición genérico-sexual de la enunciante, como porque se posiciona contrariando al discurso hegemónico de la dictadura. En este sentido, resulta necesario entender a la cultura como un campo de poderes, en palabras de Nelly Richard:

El discurso de la cultura es un campo de poderes y significaciones que entran en ejercicio desplegando una multidireccionalidad de fuerzas. Los signos que forman el lenguaje son depósitos de memorias que entremezclan varios registros en pugnas de intereses 
ideológico-culturales. Lo masculino y lo femenino son partes activas de esas controversias de significaciones que tensan los signos como materiales polémicos: como campos de batalla de producción de significados (24).

Así el discurso hegemónico de la dictadura, cuyo objetivo era castigar e invisibilizar a la oposición, tuvo un efecto particular en el sistema sexo-género, ya que la mujer militante se convirtió en una trasgresión a dicotomías tales como la mujer asociada a lo privado y el hombre a lo público. Por lo tanto, la mujer torturada era castigada no solo como "el enemigo", sino que también por haber sobrepasado el rol que la sociedad le había asignado.

Otra problemática interesante que se presenta en la novela, es el traslado que se evidencia desde el discurso marginal al hegemónico, en la medida que la protagonista que en su condición de mujer torturada era una sujeto marginal, luego pasa a formar parte del sistema hegemónico y a trabajar para él: "Hay que distraerse, muñeca. Si no, sería imposible cumplir con nuestro trabajo. Ahora somos un equipo" (Sime 66). No obstante, me parece que esta inclusión al sistema hegemónico es otra más de las estrategias utilizadas para anular al discurso marginal.

Esto último da cuenta que el surgimiento de estos discursos marginales y más aún, su relativo posicionamiento en la sociedad, es un hecho que incomoda al discurso hegemónico, ya sea el masculino o el dictatorial.

Por otra parte, debemos atender a la problemática de la escritura de mujeres, ya que ésta suele ser subvalorada frente a la literatura producida por hombres, en la medida que aún no somos capaces de reconocer que la diferencia genérico-sexual sí se manifiesta en la escritura, en palabras de Richard:

Decir que el lenguaje y la escritura son in/ diferentes a la diferencia genérico-sexual refuerza el poder establecido al seguir encubriendo las técnicas mediante las cuáles la masculinidad hegemónica disfraza con lo neutro -lo im/ personal- su manía de personalizar lo universal (34).

En consecuencia, podemos decir que la proliferación de estos discursos entendidos como marginales, permiten en primera instancia 
visibilizar su condición de secundarios en la sociedad, y a su vez, evidenciar y cuestionar las falencias de los discursos hegemónicos, entendiendo que el posicionamiento de éstos puede desbancarse y que no deben considerarse instituidos por naturaleza.

\section{Nota}

1 Licenciada en Lengua y Literatura Hispánica, con mención en literatura, U. de Chile. Estudiante del Magíster en Estudios Latinoamericanos de la misma universidad. Este artículo se enmarca en el Proyecto Fondecyt "Memoria y escritura poética de mujeres en el Cono Sur de América, 1972-2010" 1110083.

\section{Bibliografía}

Avelar, Idelber. "La práctica de la tortura y la historia de la verdad". En Nelly Richardy Alberto Moreiras (eds). Pensar en/la Postdictadura. Santiago: Cuarto Propio, 2000.

Díaz, Margarita. “Una reflexión de nuestra experiencia terapéutica a treinta años del golpe militar". En Fundación La Morada (ed). Memorias de Ocupación. Violencia sexual contra mujeres detenidas durante la dictadura. Santiago: Fundación La Morada, 2005.

Grau, Olga. "Lo erógeno herido". En Fundación La Morada (ed). Memorias de Ocupación. Violencia sexual contra mujeres detenidas durante la dictadura. Santiago: Fundación La Morada, 2005.

Gutiérrez, Paulina. "La obstinada presencia del horror". En Fundación La Morada (Ed). Memorias de Ocupación. Violencia sexual contra mujeres detenidas durante la dictadura. Santiago: Fundación La Morada, 2005.

Herrera, Carolina. "Un secreto a voces”. En Fundación La Morada (Ed). Memorias de Ocupación. Violencia sexual contra mujeres detenidas durante la dictadura. Santiago: Fundación La Morada, 2005.

Jelin, Elizabeth. Los Trabajos de la Memoria. Madrid: Siglo XXI, 2002.

------ Subjetividad y esfera pública: El género y los sentidos de familia en las memorias de la represión. (manuscrito inédito facilitado por la autora).

Moulian, Tomás. Chile: Anatomía de un mito. Santiago: LOM, 1997.

Olea, Raquel. "Cuerpo, memoria, escritura". En Nelly Richardy Alberto Moreiras (eds). Pensar en/la Postdictadura. Santiago: Cuarto Propio, 2000.

Rebolledo, Loreto. Memorias del desarraigo. Testimonios de exilio y retorno de hombres y mujeres en Chile. Santiago: Catalonia, 2006.

Richard, Nelly. Políticas y estéticas de la memoria. Santiago: Cuarto Propio, 2006.

------. Residuos y metáforas (Ensayos de crítica cultural sobre el Chile de transición) Santiago: Cuarto Propio, 1998. 
-----. "La política de los espacios, crítica cultural y debate feminista". En Richard, Nelly (ed). Masculino/Femenino. Prácticas de la diferencia y cultura democrática. Santiago: Francisco Zegers Editor, 1993.

-----. “¿Tiene sexo la escritura?” En Richard, Nelly (ed). Masculino/Femenino. Prácticas de la diferencia y cultura democrática. Santiago: Francisco Zegers Editor, 1993.

Ruiz, María Olga. "Los movimientos de la memoria". En Fundación La Morada (Ed). Memorias de Ocupación. Violencia sexual contra mujeres detenidas durante la dictadura. Santiago: Fundación La Morada, 2005.

Sime, Fátima. Carne de Perra. Santiago: LOM, 2009.

Strejilevich, Nora. El Arte de no Olvidar: Literatura Testimonial en Chile, Argentina y Uruguay entre los 80 y los 90. Buenos Aires: Editorial Catálogos, 2006. 